\title{
Study on Evolution Characteristics and Driving Types of Zengtou Village in Li County, Aba Prefecture
}

\author{
Jiangfei Du \\ College of Architecture, Southwest Minzu University, Chengdu, China \\ Email:871225313@qq.com
}

How to cite this paper: Du, J.F. (2021) Study on Evolution Characteristics and Driving Types of Zengtou Village in $\mathrm{Li}$ County, Aba Prefecture. Open Access Library Journal, 8: e7814.

https://doi.org/10.4236/oalib.1107814

Received: July 31, 2021

Accepted: August 24, 2021

Published: August 27, 2021

Copyright () 2021 by author(s) and Open Access Library Inc.

This work is licensed under the Creative Commons Attribution International License (CC BY 4.0).

http://creativecommons.org/licenses/by/4.0/

\section{(c) (i) Open Access}

\begin{abstract}
This article selects GutangQingDiaolou type traditional village head village as the study case, based on the participatory research, GIS spatial analysis, detectors and other modern technologies and methods of geography, the Ming and Qing dynasties, before and after the founding of the people to the three stages of the 80 s as the axis, discusses the thickening of the evolution of the village head and form structure, driving force and the driving mechanism. It also hopes to provide reference and thinking for other minority villages in southwest China.
\end{abstract}

\section{Subject Areas}

Architecture

\section{Keywords}

Traditional Village, Evolution Characters, Driving Type, Zengtou Village, Qiang Village

\section{Introduction}

Traditional village is the representative of China's vast countryside. It has the physical form and non-material cultuoral heritage, and has high historical, cultural, scientific, artistic, social, and economic value. As well as rural population living and production and living place for a long time, affected by natural, economic, social, cultural and other combination of multiple factors, under the people's participation, the development and evolution of traditional villages play a crucial role [1]. Therefore, it is of great significance to understand and grasp the influencing factors of the evolution of traditional villages to clear the context, 
protect and develop rural characteristics.

The complex and diverse environment of traditional villages in China leads to the complex and diverse evolution process of rural settlements. In different periods, under the influence of different backgrounds and environments, the characteristics and driving mechanisms of the evolution of traditional villages in different types of areas, different ethnic groups and different scales also vary greatly [2]. In particular, the traditional villages along the Tibetan-Qiang-Yi corridor in the mountainous areas of southwest China have small scale, scattered distribution, harsh environment and lack of natural conditions. Their formation and development in the long history are mostly a natural evolution process along with the development of social and economic levels [3]. Meanwhile, In recent years, the government's policy support, urbanization construction, protection of traditional villages, development of rural economy and implementation of rural revitalization for the minority in southwest mountainous areas are both opportunities and challenges for the traditional villages in Tibetan, Qiang and Yi ethnic areas. It is a trend of the development of traditional villages to respect nature, continue history, and fully consider the inheritance and protection of the traditional village pattern while evolving to the livability of the village. In the process of the evolution of traditional villages, due to the comprehensive effects of natural environment, social economy, policy system and other driving factors, the evolution of traditional villages presents different patterns of natural evolution, accelerated evolution and drastic evolution. The geographical location and ethnic relations of traditional villages in the corridor of Tibetan, Qiang and Yi Nationalities are quite different. So the research has a long history in the corridor of the evolution of the traditional village. Combing different economic conditions, different ethnic customs, different geographical environment, the evolution of traditional villages in different types of internal law and forming mechanism, can help us to understand and protect the different characteristics of the formation and development of ethnic village. This paper takes Zengtou village as the research object, analyzes its evolution characteristics and driving types, and puts forward the corresponding development regulation mode, so as to provide thinking for the development direction of traditional villages in the corridor of Tibetan-Qing-Yi.

\section{Zengtou Village Overview}

Zengtou Qiang village, located on the banks of Zagunao River in Li county, is the most intact Qiang architectural culture and art in the world, and still maintains the primitive Qiang village of ancient simplicity. Along the banks of the Zagu Nao River valley in the Aba Tibetan and Qiang Autonomous prefecture in Sichuan province is one of the main settlements of the Qiang people. According to historical records, their ancestors migrated from the northwest before $110 \mathrm{BC}$. In order to avoid war, they chose to live in the dangerous highlands of the mountains and breed. Zengtou village is such a long history but unknown to the 
outside world of shanzhai. Zengtou village is located in the north of Taoping Township, 10 kilometers away from the township government, and belongs to $\mathrm{Li}$ county, Aba prefecture, Sichuan province. The village east Dongshan village Luoshan group, west Tonghua Township Xishan village, south and Taoping village borders, north and Wenchuan, Heishui, Mao county connected. The river network in this village is densely distributed, with four rivers flowing through the village, namely, Heibugou, Mozigou, Hongshuigou and Baguo River. Baguo River originated in Baguo, from north to south from the village through. Zengtou village is a Qiang traditional village with a long history and distinctive features in the corridor of Tibetan, Qiang and Yi nationalities. The study on the evolution characteristics and driving types of Zengtou village can be traced back to the historical origin of Qiang traditional villages, which is representative to a certain extent.

\section{Evolution Trend and Morphological Characteristics of Village Pattern in Zengtou Village}

\subsection{Investigation and Historical Evolution of Village Dwellings in Zengtou Village}

Zengtou village has a long history. According to archaeological records, it can be traced back to the Qin and Han dynasties as early as today. Therefore, the study and analysis of the historical evolution of Zengtou village is typical and representative among Qing villages and more than 200 traditional villages in the entire corridor of Tibetan, Qiang and Yi nationality (Table 1).

Through the field survey of 148 households in Zengtou village (Figure 1), it can be seen that the dwellings in Ming and Qing dynasties and before are mainly concentrated in the upper, middle and lower villages. The small villages are located in the area with a steep slope and limited by the production and construction levels at that time, so the number of dwellings is relatively small. After the liberation in the 80s, with the constant improvement of the living and production conditions, rapid population growth, raising head village high number of local-style dwelling houses village, a small village, the village also go hand in hand, continue to increase, while the new local-style dwelling houses building by, in the big village and small village down on the trend of the transfer is not obvious, but slow to develop, in the village, has appeared on the signs; Since

Table 1. New statistics of residential dwellings in different periods in Zengtou village. (Chart source: author).

\begin{tabular}{cccccc}
\hline Place & $\begin{array}{c}\text { On, in the } \\
\text { village }\end{array}$ & $\begin{array}{c}\text { The small } \\
\text { village }\end{array}$ & $\begin{array}{c}\text { Under } \\
\text { the village }\end{array}$ & $\begin{array}{c}\text { The total number } \\
\text { of households }\end{array}$ \\
\hline Ming and Qing dynasties and before & 28 & 11 & 22 & 61 \\
From liberation to the1980s (newly added) & 9 & 11 & 12 & 32 \\
The 1980s to now (newly added) & 9 & 16 & 30 & 55 \\
The total number of households & 46 & 38 & 64 & 148 \\
\hline
\end{tabular}




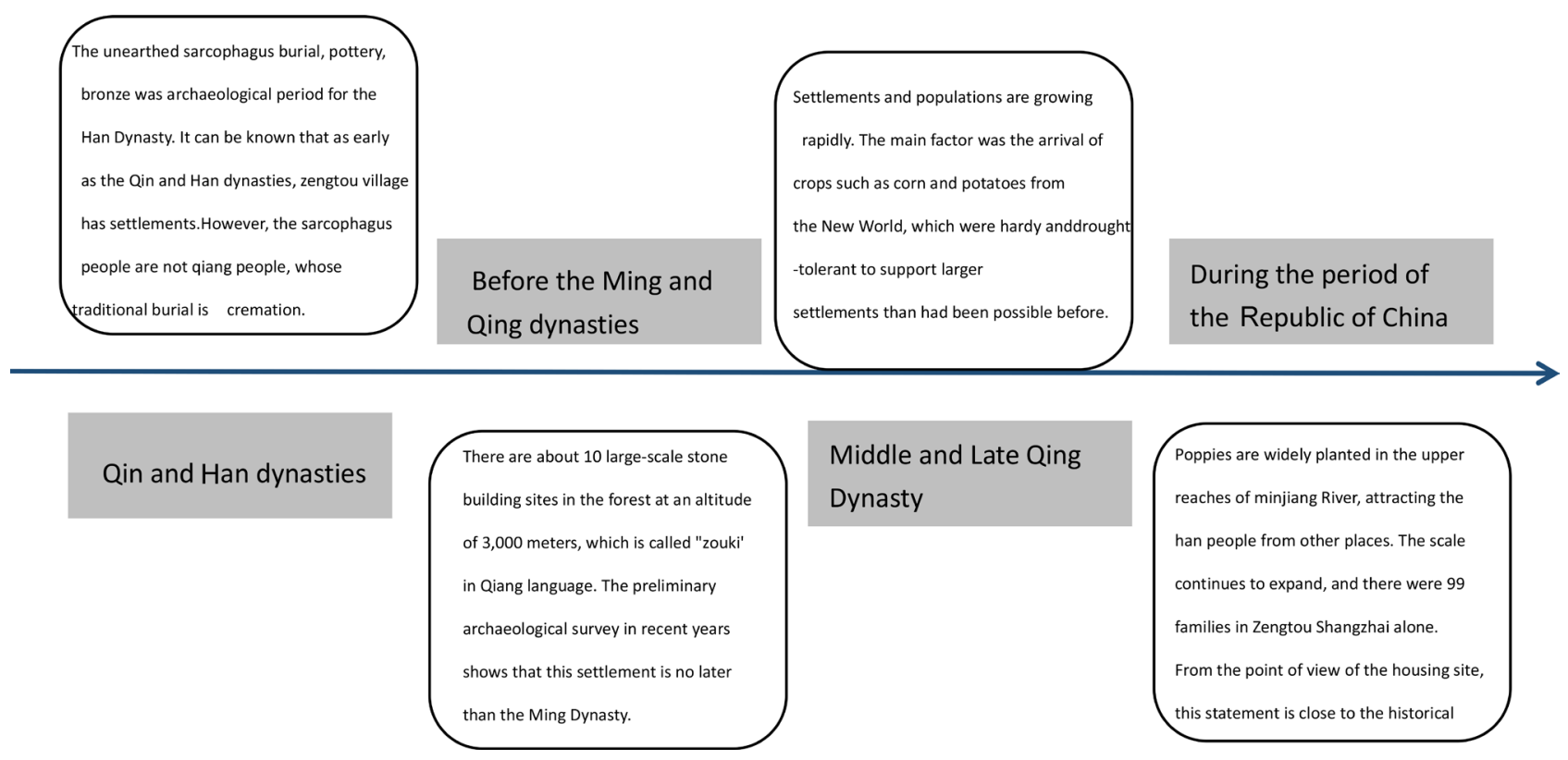

Figure 1. Historical evolution axis diagram of Zengtou village (Chart source: author).

the 1980s, driven by rural cultural tourism and other industries, the economy of Zengtou village has been developing continuously, and the new residential buildings are more inclined to road traffic, which is convenient for communication with the outside world [4]. At this time, the development of Shangzhai and Zhongzhai village is slow, while the residential buildings in Xiaozhai and Xizhai village are significantly increased, and there is still a trend of continuous expansion.

\subsection{The Evolvement Trend of Village Pattern in Zengtou Village}

The whole Qiang village in Zengtou village consists of Shangzhong village, Xiaozhai village and Xiazhai village, which are built on the gentle slope of the valley deep in the mountain. Shangzhai is the oldest part of the entire Qiang village. In ancient times, in order to defend against foreign aggression, many watchtowers were built, and the site selection was particularly exquisite (Figures 2-4). In front of the old village, close to the abyss, is Yifuguan, Wanfumo open strategic fortress; after the deep mountains and forests, so that the enemy did not dare to easily over, can be said to have a retreat. The village is located in an old forest area, surrounded by a thousand hectares of reclaimed terraced fields, enough to satisfy the daily life of the villagers during the war.

Origin (before Ming and Qing dynasties): The three houses of Wang and Zhou formed the Qiang tribe group. In order to meet the needs of defense [5], the original Shangzhai village was far away from Morzi Valley (river), close to the slope of the mountain, backed by woodland, and the residential buildings were closely gathered around the Diaolou (Figure 5).

Development (post-liberation to the 1980s): Gradually form 11 towers centered on, in, under, small four village system, most of the local-style dwelling 


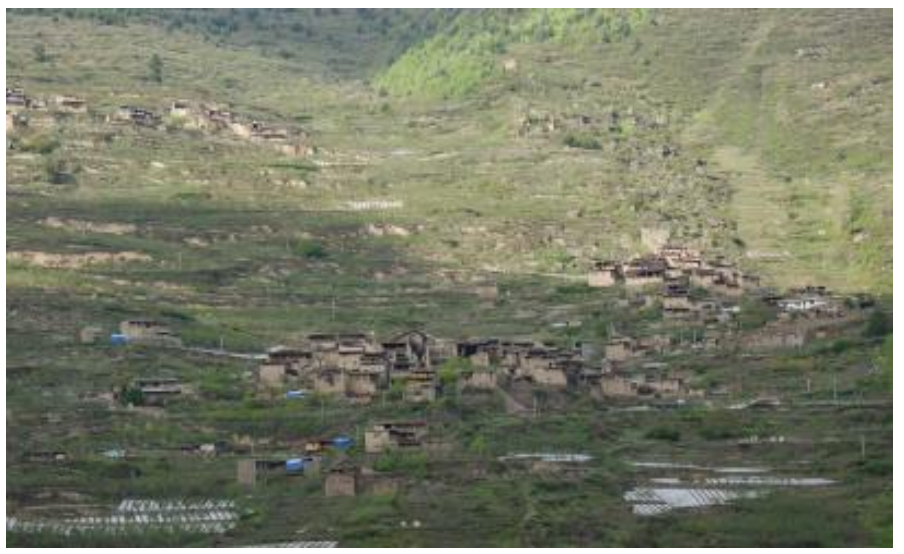

Figure 2. Overall view of the stockade.

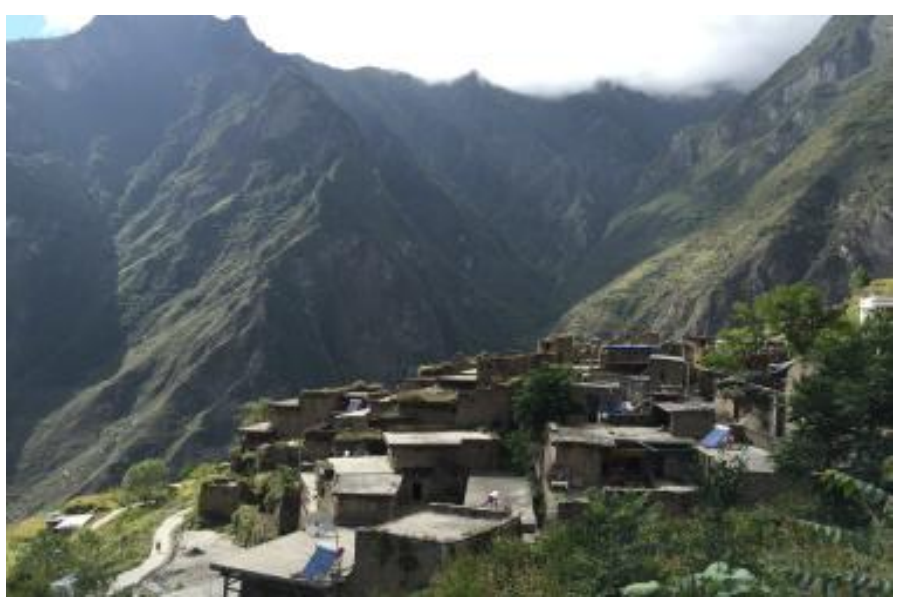

Figure 3. Overview of Shangzhai.

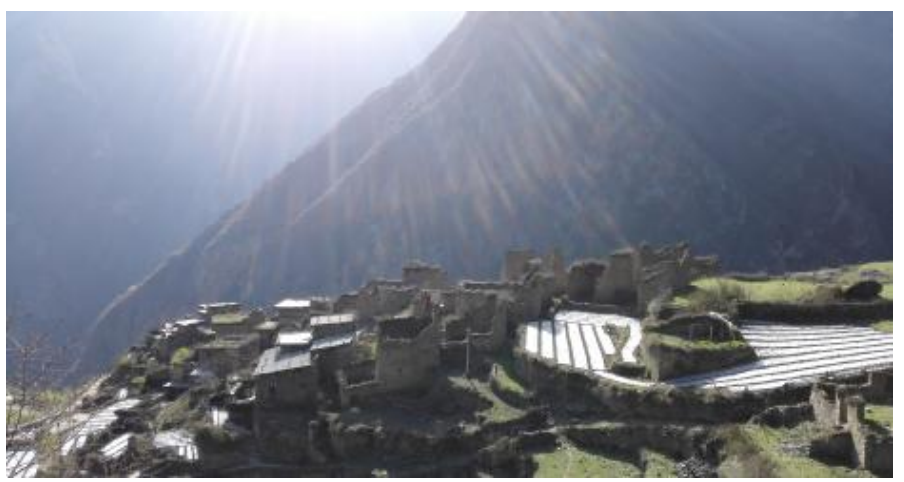

Figure 4. Overall view of the lower village.

houses building still along the Diaolou distribution, but the trend distribution along the road, the village on the expansion of basic stagnant, the village and the village in the trend of rapid expansion, while a small portion of the scattered dwellings of cultivated land and the surrounding roads, convenient lines and farming (Figure 6).

Evolution up to now (the 1980s to now): The development of Shangzhai and Zhongzhai is basically at a standstill, with only a small number of new buildings 
appearing in their periphery, while Xiazhai and Zhongzhai have relatively explosive development, with a pattern of expansion along the road. Therefore, the development trend of the upper, middle, lower and small villages is obvious, and the spatial form and architectural texture of Zengtou village are basically finalized (Figure 7).

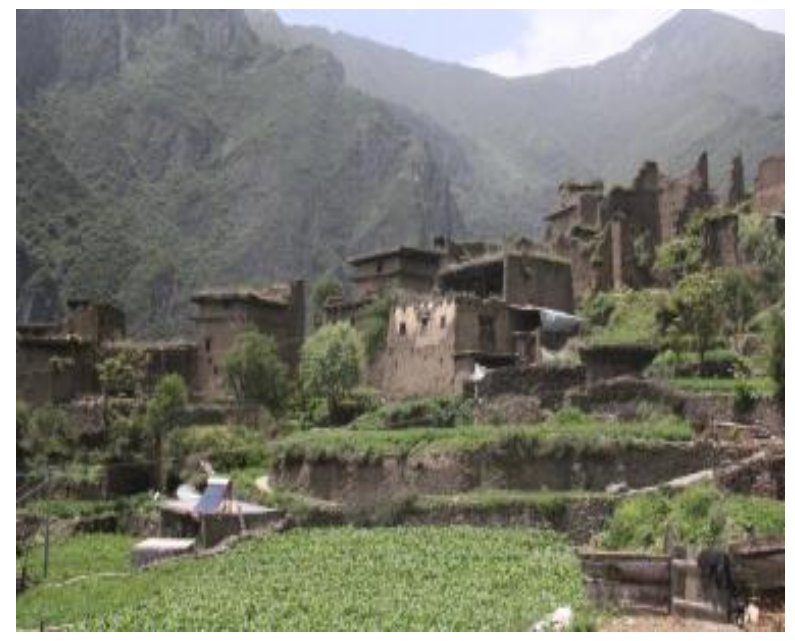

Figure 5. Residential buildings in Ming and Qing dynasties and before.

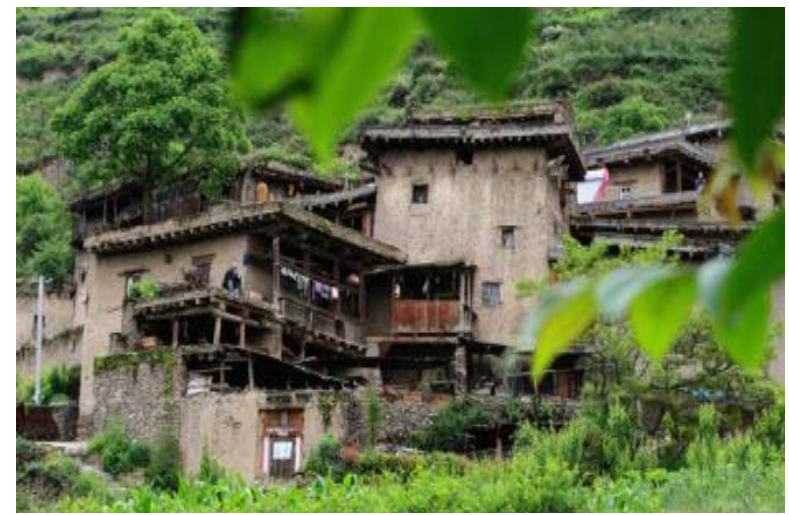

Figure 6. Residential buildings from liberation to the 1980s.

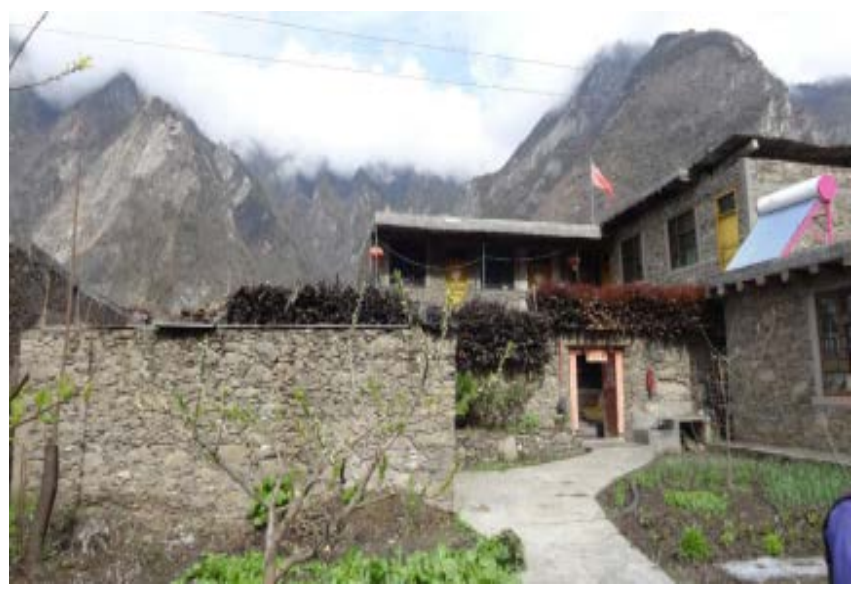

Figure 7. Residential buildings from the 1980s to now. 
By increasing the origin of the first village's development, so far we can work out the originated in the small village and the main village development, finalize the design in the next village; its development also convert the defensive starting with the most convenient farming life, thus it can be seen that with the passage of time and the different social background, life ideas of the villagers, profound changes have taken place, It then influences the evolution and development trend of the village (Table 2 ).

\subsection{Study on the Evolution Characteristics of the Overall Spatial Morphology of Zengtou Village}

\section{1) Morphological evolution of the outer boundary of Zengtou village}

Through field investigation, comparison of high-definition satellite images and collection of a large number of relevant data, it can be seen that the boundary of Zengtou village is a mixed boundary, and the upper, middle and lower three Zhaizhu villages are artificial boundary and natural boundary interaction. In the northeast of Shangzhai, the elevation is too high, forming a natural barrier boundary. Zhongzhai is mainly distributed along roads, forming artificial boundaries. Xiazhai develops in the northeast and northwest directions and is mainly distributed on both sides of the road and in the valley area, which belongs to the mixed boundary (Figure 8).

\section{2) The evolution of Zengtou village development center}

In the Ming and Qing dynasties and before, the development center was not obvious, the upper, middle and lower villages had not yet formed the scale, the three villages appeared according to local conditions of sheet distribution, at the same time, the Diaolou as the center to spread around the distribution, the center of the three villages are in the relatively inward region; From the founding of the People's Republic of China to the 1980s, the three pillars of Shangzhai, Zhongzhai and Xiachai had changed. Shangzhai was in a relatively stagnant state, and

Table 2. Chronological analysis and evolution trend diagram of Zengltou village.
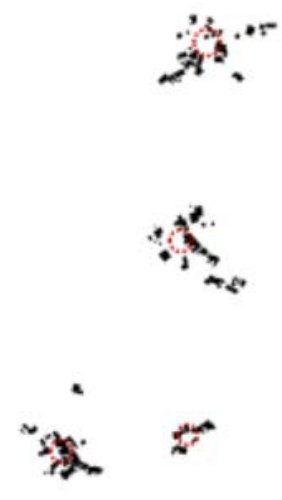

Ming and Qing dynasties and before From liberation to the 1980 s
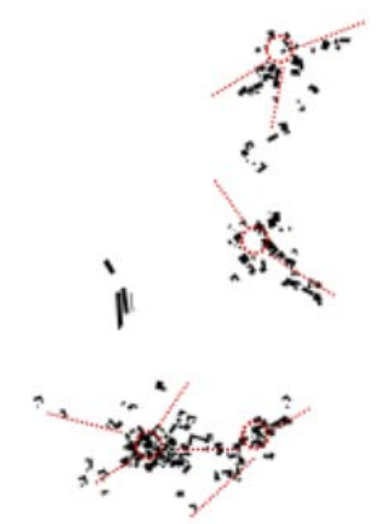


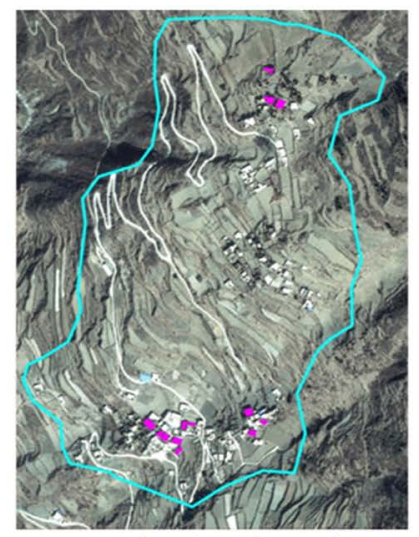

During the Ming and Qing dynasties

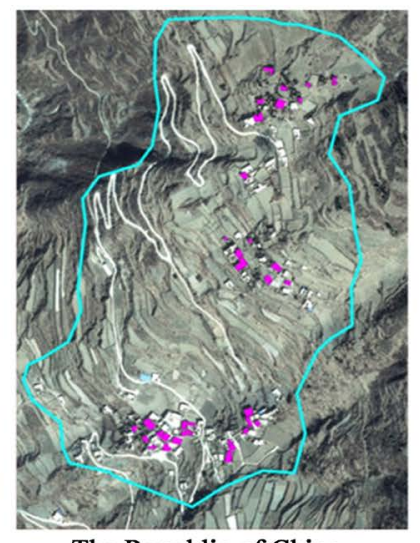

The Republic of China to liberation

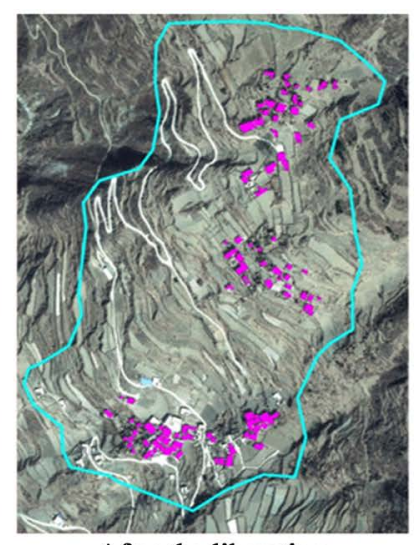

After the liberation

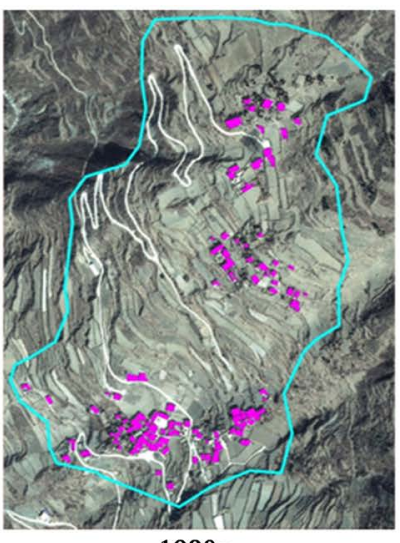

1980s

Figure 8. Boundary morphology evolution of Zengtou village (Photo source: Zengtou village protection planning).

Zhongzhai and Xiachai had not broken the diffusion pattern from the center to the surrounding area, but there was also a situation of sparse diffusion to a certain direction. Since the 1980s, Shangzhai and Zhongzhai have been stagnating, while Xiachai has made great progress, but its center has also been shifting to the northeast. It can be seen from this that, under the influence of modern social thoughts, the change of functional structure such as production and living has a significant impact on the spatial structure of villages. The development center of Zengtou village has been transferred successively in the upper, middle and lower villages, reflecting the importance of agricultural production and convenient travel for villagers, and the villagers have also shifted from defense to open development.

\section{Driving Force Analysis of Village Pattern in Zengtou Village}

\subsection{Natural Environment and Feng Shui Pattern}

Negative Yin hug Yang, back mountain face water north according to the ridge, west melt landscape, east farmland, south river, looking at the case mountain. The landscape pattern of Zengtou village perfectly fits the traditional feng shui concept. The overall topography of the village is high in the north and low in the east. It is located on the mountainside and dominates. Depending on the topography and landform, the defense space between the villages and the terrace living space is formed.

Raise head village, rows, and wooden house built up to the mountain village with pure ancient Qiang folk houses, more unique "three ridge slope two water pattern, which is traditional village with a history of accumulation, is a reflection of traditional culture, through structure constitute the complete integral pattern, determine the planning and the overall framework of pattern for: "three mountain two water, three-four settlements". In Ming and Qing dynasties and before, villages were irregular in the distribution of each village area. With historical changes, the following villages were gradually selected as the development center 
for more suitable development, and they were distributed along the valley and road. However, no matter how the villages developed, they did not break away from the pattern of "three mountains and two rivers".

\subsection{Cultural Concept}

The layout of Zengtou Qiang's "three mountains, two rivers, three areas and four settlements" is not only restricted by the high mountains and valleys but also influenced by the Qiang's own reverence and belief in nature. The Qiang people worship the nature, believe in nature, take measures according to local conditions, choose to live, and seek their own living space on the basis of the belief in nature. From the village pattern to the architectural form and material, they fully integrate and adapt to the local natural environment, and conform to the terrain.

\subsection{Defense Needs}

At first, Zengtou Qiang village built the buildings at a high altitude, and presented the distribution of multiple groups, each group's boundary is relatively compact, most of the residential buildings are distributed with the Diaolou as the center. With social changes, conflicts between ethnic groups in western Sichuan gradually decrease, and life gradually develops toward harmony. The defense needs of Qiang traditional villages gradually weaken, and the residential houses begin to disperse along the roads. The overall spatial texture of the villages presents a more diverse, open and inclusive trend [6].

\section{Conclusions}

In this paper, participatory research, GIS spatial analysis, geographic detector and other modern technologies and methods were used to conduct an in-depth study on the evolution characteristics and driving types of Zengtou village, and the driving forces of the village pattern were analyzed in combination with the regional characteristics of the Qiang ethnic group, and the following conclusions were drawn: 1) With the passage of time and the difference of social background, the life concept of the villagers has also undergone profound changes, which then affects the evolution and development trend of the village; 2) Although the layout of Qiang traditional villages has changed in the evolution process, Zengtou village does not break away from the pattern of "three mountains and two rivers". On the surface, Qiang people still have a strong concept of feng shui and nature worship.

The evolution of the pattern of traditional villages is comprehensively influenced by various factors and behavioral subjects in different periods. In the later period, the internal laws and influencing mechanisms of the growth of traditional villages can be quantitatively analyzed, so as to make a scientific judgment on the evolution characteristics and driving types in a certain period. It provides some research ideas on the evolution characteristics and driving types of other 
traditional villages in the corridor of Tibetan-Qing-Yi.

\section{Conflicts of Interest}

The author declares no conflicts of interest.

\section{References}

[1] Huang, Y.C. (2018) Study on the Morphological Evolution of Zhangzhou Traditional Villages under the Intersection of Multiple Cultures. Doctoral Dissertation, Hunan University, Changsha.

[2] Li, Y.B., et al. (2018) Evolution and Driving Mechanism of Villages in Typical Peak Cluster Depressions in Guizhou Province in Recent 50 Years. Agricultural Resources and Regional Planning in China, 38, 32-36.

[3] Yao, L., Li, X.K., et al. (2020) Spatial Evolution Characteristics and Driving Mechanisms of Villages in Poor Areas of Southwest China. Journal of Ningxia Normal University, 41, 22-26.

[4] Zhu, S.S. (2020) Rural Spatial Evolution Driven by "Capital Going to the Countryside": A Comparative Case Study Based on Three Typical Villages in North Zhejiang. Enterprise Economic, 37, 12-15.

[5] Lai, Y.J., Yang, M.Y., et al. (2019) Quantitative Study on Spatial Morphology Evolution of Qiang Traditional Villages: Taking Taoping Qiang Village as an Example. Architecture Forum and Architecture Design, 39, 41-45.

[6] Zhang, Z.Q., Tan, Y.M., et al. (2017) Study on the Morphological Evolution of Traditional Villages Based on Settlement Dynamic Evolution Theory: A Case Study of Shanbei Village in Western Hunan Province. Areal Research and Development, 32, $38-42$. 